Proyecciones Journal of Mathematics

Vol. 35, No 2, pp. 197-211, June 2016.

Universidad Católica del Norte

Antofagasta - Chile

\title{
Parametric Estimation and the CIR Model *
}

\author{
Eugenio Saavedra \\ Universidad de Santiago de Chile, Chile \\ Received: January 2014. Accepted : March 2016
}

\begin{abstract}
We study parametric estimation in the Cox-Ingersoll-Ross model and establish the stochastic differential equations for the parameters involved in it.
\end{abstract}

Keywords: estimating function, quasi-likelihood, stochastic differential equation.

AMS Classification : 60H10, 62P20, 62M99.

${ }^{*}$ Part of this work was supported by DICYT Grant \# 049833SG 


\section{Introduction}

We consider the dynamics (unidimensional)

(1.1) $d X_{t}=\mu\left(t, X_{t}, \theta_{t}\right) d t+\sigma\left(t, X_{t}, \theta_{t}\right) d W_{t} \quad X_{0}=x_{0}, \quad 0 \leq T<\infty$,

where $\theta_{t}=\left(\alpha_{t}, \beta_{t}, \sigma_{t}\right), \mu\left(t, X_{t}, \theta_{t}\right)=\alpha_{t} a\left(X_{t}\right)+\beta_{t} b\left(X_{t}\right), \sigma\left(t, X_{t}, \theta_{t}\right)=$ $\sigma_{t} X_{t}^{p+1}$ and $W=\left\{W_{t} ; \quad 0 \leq t<\infty\right\}$ is a standard Wiener process on a given probability space $(\Omega, F, P)$. We assume that the drift and diffusion coefficients are functions that satisfy technical conditions which guarantee that equation (1.1) has a unique strong solution.

The object of this article is to estimate $\theta$ in the Cox-Ingersoll-Ross model, denoted CIR model, which is a particular case of equation (1.1) and widely used in financial literature to model interest rates.

In Section 2, we introduce two types of changes of variables which reduce equation (1.1) to equivalent equations, though with additive noise. In each case we find the estimator of $\theta$ when it is a constant parameter. Section 3 contains our main result. In the CIR model, we establish the stochastic differential equation that the estimator of $\theta$ must satisfy.

\section{Quasi-Likelihood estimators for a class of diffusion pro- cesses}

We assume that $\theta_{t}$ is constant. In order to allow for a convenient estimation methodology, we define the time transformation

$$
\begin{aligned}
\rho(t) & =\int_{0}^{t} \sigma^{2}\left(s, X_{s}, \theta\right) d s \\
& =\langle X\rangle_{t} \\
& =\sigma^{2} \int_{0}^{t} X_{s}^{2(p+1)} d s
\end{aligned}
$$

where $\langle X\rangle$ denotes the quadratic variation of $X$.

We set

$$
Y_{\rho(t)}=X_{t}
$$

Then equation (1.1) is transformed into the dynamics with additive noise

$$
d Y_{\rho}=\frac{\mu\left(\rho, Y_{\rho}, \theta\right)}{\sigma^{2}\left(\rho, Y_{\rho}, \theta\right)} d \rho+d \tilde{W}_{\rho}, \quad Y_{0}=x_{0}
$$




$$
=\left(\frac{1}{\sigma^{2}} \alpha a\left(Y_{\rho}\right) Y_{\rho}^{-2(p+1)}+\frac{1}{\sigma^{2}} \beta b\left(Y_{\rho}\right) Y_{\rho}^{-2(p+1)}\right) d \rho+d \tilde{W}_{\rho},
$$

for all $0 \leq \rho \leq \rho(T)$, where $\tilde{W}=\left\{\tilde{W}_{\rho} ; 0 \leq \rho<\infty\right\}$ is a standard Wiener process with respect to time $\rho$ (see Feigin, 1976).

In addition, the nature of the diffusion processes allows us to evaluate $\sigma^{2}$ with probability one on the basis of knowledge of a path of the process on any finite time interval. This follows from the definitions of the quadratic variation process and stochastic integrals with respect to the Wiener process from which we obtain

$$
\sigma^{2}=\frac{\lim _{\left\|\sigma_{n}\right\| \downarrow 0} \sum_{i=0}^{k_{n}}\left(X_{t_{i+1}^{(n)}}-X_{t_{i}^{(n)}}\right)^{2}}{\lim _{\left\|\sigma_{n}\right\| \downarrow 0} \sum_{i=0}^{k_{n}} X_{t_{i+1}^{(n)}}^{2(p+1)}\left(t_{i+1}^{(n)}-t_{i}^{(n)}\right)}
$$

where $\sigma_{n}$ denotes the partition $0=t_{0}^{(n)}<t_{1}^{(n)}<\cdots<t_{k_{n}}^{(n)}=T$ and $\left\|\sigma_{n}\right\|==\max _{0 \leq i \leq k_{n-1}} t_{i+1}^{(n)}-t_{i}^{(n)}$.

Therefore we may consider that the parameter $\sigma$ is known and assume that the parameter $p$ is also known. Thus we now concentrate on the estimation of the parameter $\theta=(\alpha, \beta)$.

According to Heyde (1997), for both the dynamics (1.1) and (2.3) the quasi-likelihood approach yields the respective estimating equations for the parameter $\theta$, namely,

$$
\begin{aligned}
& \int_{0}^{T} a\left(X_{t}\right) X_{t}^{-2(p+1)}\left[d X_{t}-\left(\alpha a\left(X_{t}\right)+\beta b\left(X_{t}\right)\right) d t\right]=0 \\
& \int_{0}^{T} b\left(X_{t}\right) X_{t}^{-2(p+1)}\left[d X_{t}-\left(\alpha a\left(X_{t}\right)+\beta b\left(X_{t}\right)\right) d t\right]=0
\end{aligned}
$$

and

$$
\begin{gathered}
\int_{0}^{\sigma(T)} a\left(Y_{\rho}\right) Y_{\rho}^{-2(p+1)}\left[d Y_{\rho}-\left(\frac{1}{\sigma^{2}} \alpha a\left(Y_{\rho}\right) Y_{\rho}^{-2(p+1)}+\frac{1}{\sigma^{2}} \beta b\left(Y_{\rho}\right) Y_{\rho}^{-2(p+1)}\right) d \rho\right] \\
=0
\end{gathered}
$$


$\int_{0}^{\sigma(T)} b\left(Y_{\rho}\right) Y_{\rho}^{-2(p+1)}\left[d Y_{\rho}-\left(\frac{1}{\sigma^{2}} \alpha a\left(Y_{\rho}\right) Y_{\rho}^{-2(p+1)}+\frac{1}{\sigma^{2}} \beta b\left(Y_{\rho}\right) Y_{\rho}^{-2(p+1)}\right) d \rho\right]=0$

We set

$$
I(n, S, U, f)=\int_{0}^{S} f\left(U_{\rho}\right) U_{\rho}^{-n(p+1)} d \rho
$$

and

$$
J(n, T, U, f)=\int_{0}^{S} f\left(U_{\rho}\right) U_{\rho}^{-n(p+1)} d U_{\rho}
$$

By (2.6), the quasi-likelihood estimators $\hat{\alpha}_{1, T}$ and $\hat{\beta}_{1, T}$ are given by

$$
\hat{\alpha}_{1, T}=\sigma^{2} \frac{J(2, \rho(T), Y, a) I\left(4, \rho(T), Y, b^{2}\right)-I(2, \rho(T), Y, b) I(4, \rho(T), Y, a b)}{I\left(4, \rho(T), Y, a^{2}\right) I\left(4, \rho(T), Y, b^{2}\right)-I^{2}(4, \rho(T), Y, a b)}
$$

$$
\hat{\beta}_{1, T}=\sigma^{2} \frac{J(2, \rho(T), Y, b) I\left(4, \rho(T), Y, a^{2}\right)-J(2, \rho(T), Y, a) I(4, \rho(T), Y, a b)}{I\left(4, \rho(T), Y, a^{2}\right) I\left(4, \rho(T), Y, b^{2}\right)-I^{2}(4, \rho(T), Y, a b)}
$$

When $a \equiv 0$ or $\mathrm{b} \equiv 0$, say $b \equiv 0$, we have

$$
\hat{\alpha}_{1, T}=\frac{J(2, \rho(T), Y, a)}{I\left(4, \rho(T), Y, a^{2}\right)} .
$$

Analogously, the next two equations

$$
\begin{gathered}
\hat{\alpha}_{2, T}=\frac{J(2, T, X, a) I\left(4, T, X, b^{2}\right)-I(2, T, X, a b) J(2, T, X, b)}{I\left(2, T, X, a^{2}\right) I\left(2, T, X, b^{2}\right)-I^{2}(2, T, X, a b)} \\
\hat{\beta}_{2, T}=\frac{J(2, T, X, b) I\left(2, T, X, a^{2}\right)-J(2, T, X, a) I(2, T, X, a b)}{I\left(2, T, X, a^{2}\right) I\left(2, T, X, b^{2}\right)-I^{2}(2, T, X, a b)}
\end{gathered}
$$

follow from $(2.5)$. 
Now when $b \equiv 0$ we have

$$
\hat{\alpha}_{2, T}=\frac{J(2, T, X, a)}{I\left(2, T, X, a^{2}\right)} .
$$

We again consider equation (1.1). Our next result is an alternate approach for estimating the parameter $(\alpha, \beta)$.

Lemma 1. Let $Z$ be the solution of the stochastic differential equation

$$
\begin{aligned}
d Z_{\tau}= & {\left[\alpha a\left(Z_{\tau}^{-\frac{1}{p}}\right)\left(-\frac{1}{p \sigma^{2}}\right) Z_{\tau}^{1+\frac{1}{p}}+\beta b\left(Z_{\tau}^{-\frac{1}{p}}\right)\left(-\frac{1}{p \sigma^{2}}\right) Z_{\tau}^{1+\frac{1}{p}}\right.} \\
& \left.+\frac{1}{2}\left(1+\frac{1}{p}\right) Z_{\tau}^{-1}\right] d \tau+d \hat{W}_{\tau}, \\
Z_{0}= & x_{0}^{-p}, \quad 0 \leq \tau \leq \tilde{T}=\sigma^{2} p^{2} T .
\end{aligned}
$$

We set

$$
K(Z, S, f)=\frac{1}{2}\left(1+\frac{1}{p}\right) \int_{0}^{S} f\left(Z_{s}^{-\frac{1}{p}}\right) Z_{s}^{\frac{1}{p}} d s-\int_{0}^{S} f\left(Z_{s}^{-\frac{1}{p}}\right) Z_{s}^{1+\frac{1}{p}} d Z_{s} .
$$

Then the quasi-likelihood estimators of $\alpha$ and $\beta$, denoted respectively $\hat{\alpha}_{3, T}$ and $\hat{\beta}_{3, T}$, are given by

$$
\hat{\alpha}_{3, T}=\sigma^{2} p \frac{K(Z, \tilde{T}, a) I\left(2, \tilde{T}, Z^{-\frac{1}{p}}, b^{2}\right)-K(Z, \tilde{T}, b) I\left(2, \tilde{T}, Z^{-\frac{1}{p}}, a b\right)}{I\left(2, \tilde{T}, Z^{-\frac{1}{p}}, a^{2}\right) I\left(2, \tilde{T}, Z^{-\frac{1}{p}}, b^{2}\right)-I^{2}\left(2, \tilde{T}, Z^{-\frac{1}{p}}, a b\right)}
$$

$$
\hat{\beta}_{3, T}=\sigma^{2} p \frac{K(Z, \tilde{T}, b) I\left(2, \tilde{T}, Z^{-\frac{1}{p}}, a^{2}\right)-K(Z, \tilde{T}, a) I\left(2, \tilde{T}, Z^{-\frac{1}{p}}, a b\right)}{I\left(2, \tilde{T}, Z^{-\frac{1}{p}}, a^{2}\right) I\left(2, \tilde{T}, Z^{-\frac{1}{p}}, b^{2}\right)-I^{2}\left(2, \tilde{T}, Z^{-\frac{1}{p}}, a b\right)}
$$


When $a \equiv 0$ or $b \equiv 0, \quad$ say $b \equiv 0$, we obtain

$$
\hat{\alpha}_{3, T}=\sigma^{2} p \frac{K(Z, \tilde{T}, a)}{I\left(2, \tilde{T}, Z^{-\frac{1}{p}}, a^{2}\right)} .
$$

Proof. Let $H_{t}$ be the stochastic process

$$
H_{t}=X_{t}^{-p}, \quad p \neq 0, \text { for all } 0 \leq t<\infty .
$$

By Ito's formula,

$d H_{t}=\left[\alpha a\left(H_{t}^{-\frac{1}{p}}\right)(-p) H_{t}^{1+\frac{1}{p}}+\beta b\left(H_{t}^{-\frac{1}{p}}\right)(-p) H_{t}^{1+\frac{1}{p}}++\frac{1}{2} \sigma^{2} p(p+1) H_{t}^{-1}\right] d t+\sigma(-p) d W_{t}$.

Furthermore, we define a time transformation by

$$
\tau(t)=\sigma^{2} p^{2} t \quad, \quad 0 \leq t \leq T,
$$

and set $Z_{\tau(t)}=H_{t}$.

Then

$$
\begin{aligned}
d Z_{\tau}= & {\left[\alpha a\left(Z_{\tau}^{-\frac{1}{p}}\right)\left(-\frac{1}{p \sigma^{2}}\right) Z_{\tau}^{1+\frac{1}{p}}+\beta b\left(Z_{\tau}^{-\frac{1}{p}}\right)\left(-\frac{1}{p \sigma^{2}}\right) Z_{\tau}^{1+\frac{1}{p}}\right.} \\
& \left.+\frac{1}{2}\left(1+\frac{1}{p}\right) Z_{\tau}^{-1}\right] d \tau+d \hat{W}_{\tau} \\
Z_{0}= & x_{0}^{-p}, \quad 0 \leq \tau \leq \tau(T)=\tilde{T}
\end{aligned}
$$

For dynamics (2.17) obtain the estimating equation

$$
\int_{0}^{\tilde{T}}\left(\begin{array}{c}
Z_{\tau}^{1+\frac{1}{p}} a\left(Z_{\tau}^{-\frac{1}{p}}\right) \\
Z_{\tau}^{1+\frac{1}{p}} b\left(Z_{\tau}^{-\frac{1}{p}}\right)
\end{array}\right)\left[d Z_{\tau}-\left(\alpha a\left(Z_{\tau}^{-\frac{1}{p}}\right)\left(\frac{-1}{p \sigma^{2}}\right) Z_{\tau}^{1+\frac{1}{p}}\right.\right.
$$

$$
\left.\left.+\beta b\left(Z_{\tau}^{-\frac{1}{p}}\right)\left(\frac{-1}{p \sigma^{2}}\right) Z_{\tau}^{1+\frac{1}{p}}+\frac{1}{2}\left(1+\frac{1}{p}\right) Z_{\tau}^{-1}\right) d \tau\right]=\left(\begin{array}{l}
0 \\
0
\end{array}\right) .
$$


The proof now follows by solving this equation.

\section{Example: The Cox-Ingersoll-Ross Model}

Consider the stochastic differential equation

$$
d X_{t}=\theta_{1}\left(\theta_{2}-X_{t}\right) d t+\sigma \sqrt{X_{t}} d W_{t}, X_{0}=x_{0}, 0 \leq t \leq T
$$

where $x>0, \theta_{1}>0, \theta_{2}>0$ and $\sigma>0$. In financial literature, processes satisfying (2.19) are often referred to as mean reverting processes. The parameter $\theta_{2}$ is the mean reversion level and the parameter $\theta_{1}$ the speed of adjustment.

The drift and diffusion coefficients may be written as (1.1), with $\alpha_{t}=\theta_{1} \theta_{2}, \beta_{t}=-\theta_{1}, \sigma_{t}=\sigma, a(x)=1, b(x)=x$ and $p=-\frac{1}{2}$.

Thus, in this example, equations (2.1), (2.3), (2.16) and (2.17) have the respective forms:

$$
\rho(t)=\sigma^{2} \int_{0}^{t} X_{s} d s
$$

$$
\begin{gathered}
d Y_{t}=\left(\frac{1}{\sigma^{2}} Y_{t}^{-1} \alpha+\frac{1}{\sigma^{2}} \beta\right) d t+d \tilde{W}_{t}, Y_{0}=x_{0}, 0 \leq t \leq \rho(T), \\
\tau(t)=\frac{\sigma^{2}}{4} t \quad, \quad \tilde{T}=\frac{\sigma^{2}}{4} T, \\
d Z_{t}=\left[\left(\frac{2}{\sigma^{2}} \alpha-\frac{1}{2}\right) Z_{t}^{-1}+\frac{2}{\sigma^{2}} \beta Z_{t}\right] d t+d \hat{W}_{t}, Z_{0}=\sqrt{x_{0}}, 0 \leq t \leq \tilde{T} .
\end{gathered}
$$

To estimate parameters $\alpha$ and $\beta$, we apply formulas (2.7), (2.9) and (2.12) to obtain

$$
\begin{aligned}
& (2.24) \hat{\alpha}_{1, T}=\sigma^{2} \frac{\rho(T)\left(\int_{0}^{\rho(T)} Y_{s}^{-1} d Y_{s}\right)-\left(Y_{\rho(T)}-Y_{0}\right)\left(\int_{0}^{\rho(T)} Y_{s}^{-1} d_{s}\right)}{\rho(T)\left(\int_{0}^{\rho(T)} Y_{s}^{-2} d s\right)-\left(\int_{0}^{\rho(T)} Y_{s}^{-1} d s\right)^{2}} \\
& =\sigma^{2} \frac{\rho(T)\left(\log \left[\frac{Y_{\rho(T)}}{Y_{0}}\right]+\frac{1}{2} \int_{0}^{\rho(T)} Y_{s}^{-2} d s\right)-\left(Y_{\rho(T)}-Y_{0}\right)\left(\int_{0}^{\rho(T)} Y_{s}^{-1} d s\right)}{\rho(T)\left(\int_{0}^{\rho(T)} Y_{s}^{-2} d s\right)-\left(\int_{0}^{\rho(T)} Y_{s}^{-1} d s\right)^{2}},
\end{aligned}
$$




$$
\begin{gathered}
\hat{\alpha}_{2, T}=\frac{\left(\int_{0}^{T} X_{s}^{-1} d X_{s}\right)\left(\int_{0}^{T} X_{s} d s\right)-\left(X_{T}-X_{0}\right) T}{\left(\int_{0}^{T} X_{s}^{-1} d s\right)\left(\int_{0}^{T} X_{s} d s\right)-T^{2}} \\
=\frac{\left(\log \left[\frac{X_{T}}{X_{0}}\right]+\frac{1}{2} \int_{0}^{T} X_{s}^{-2} d s\right)\left(\int_{0}^{T} X_{s} d s\right)-\left(X_{T}-X_{0}\right) T}{\left(\int_{0}^{T} X_{s}^{-1} d s\right)\left(\int_{0}^{T} X_{s} d s\right)-T^{2}}
\end{gathered}
$$

$$
\begin{aligned}
\hat{\alpha}_{3, T}= & \frac{\sigma^{2}}{2}\left[\frac{\frac{1}{2}\left(\int_{0}^{\tilde{T}} Z_{s}^{2} d s\right)\left(\int_{0}^{\tilde{T}} Z_{s}^{-2} d s\right)+\left(\int_{0}^{\tilde{T}} Z_{s}^{2} d s\right)\left(\int_{0}^{\tilde{T}} Z_{s}^{-1} d Z_{s}\right)}{\left(\int_{0}^{\tilde{T}} Z_{s}^{-2} d s\right)\left(\int_{0}^{\tilde{T}} Z_{s}^{2} d s\right)-\tilde{T}^{2}}\right. \\
& \left.-\frac{\frac{1}{2} \tilde{T}^{2}+\tilde{T}\left(\int_{0}^{\tilde{T}} Z_{s} d Z_{s}\right)}{\left(\int_{0}^{\tilde{T}} Z_{s}^{-2} d s\right)\left(\int_{0}^{\tilde{T}} Z_{s}^{2} d s\right)-\tilde{T}^{2}}\right] \\
= & \frac{\sigma^{2}}{2}\left[\frac{1}{2}+\frac{\left(\int_{0}^{\tilde{T}} Z_{s}^{2} d s\right)\left(\int_{0}^{\tilde{T}} Z_{s}^{-1} d Z_{s}\right)-\tilde{T}\left(\int_{0}^{\tilde{T}} Z_{s} d Z_{s}\right)}{\left(\int_{0}^{\tilde{T}} Z_{s}^{-2} d s\right)\left(\int_{0}^{\tilde{T}} Z_{s}^{2} d s\right)-\tilde{T}^{2}}\right],
\end{aligned}
$$

and

$$
\hat{\beta}_{1, T}=\sigma^{2} \frac{-\left(\int_{0}^{\rho(T)} Y_{s}^{-1} d Y_{s}\right)\left(\int_{0}^{\rho(T)} Y_{s}^{-1} d s\right)+\left(Y_{\rho(T)}-Y_{0}\right)\left(\int_{0}^{\rho(T)} Y_{s}^{-2} d s\right)}{\rho(T)\left(\int_{0}^{\rho(T)} Y_{s}^{-2} d s\right)-\left(\int_{0}^{\rho(T)} Y_{s}^{-1} d s\right)^{2}}
$$

$$
\begin{gathered}
=\sigma^{2} \frac{-\left(\int_{0}^{\rho(T)} Y_{s}^{-1} d s\right)\left(\log \left[\frac{Y_{\rho(T)}}{Y_{0}}\right]+\frac{1}{2} \int_{0}^{\rho(T)} Y_{s}^{-2} d s\right)+\left(Y_{\rho(T)}-Y_{0}\right)\left(\int_{0}^{\rho(T)} Y_{s}^{-2} d s\right)}{\rho(T)\left(\int_{0}^{\rho(T)} Y_{s}^{-2} d s\right)-\left(\int_{0}^{\rho(T)} Y_{s}^{-1} d s\right)^{2}}, \\
\hat{\beta}_{2, T}=\frac{\left(\int_{0}^{T} X_{s}^{-1} d s\right)\left(X_{T}-X_{0}\right)-\left(\int_{0}^{T} X_{s}^{-1} d X_{s}\right) T}{\left(\int_{0}^{T} X_{s}^{-1} d s\right)\left(\int_{0}^{T} X_{s} d s\right)-T^{2}}
\end{gathered}
$$

$$
\begin{aligned}
&= \frac{\left(\int_{0}^{T} X_{s}-1 d s\right)\left(X_{T}-X_{0}\right)\left(\left(\log \left[\frac{X_{T}}{X_{0}}\right]+\frac{1}{2} \int_{0}^{T} X_{s}^{-2} d s\right) T\right.}{\left(\int_{0}^{T} X_{s}^{-1} d s\right)\left(\int_{0}^{T} X_{s} d s\right)-T^{2}}, \\
& \hat{\beta}_{3, T}=\frac{\sigma^{2}}{2} \frac{\left(\int_{0}^{\tilde{T}} Z_{s}^{-2} d s\right)\left(\int_{0}^{\tilde{T}} Z_{s} d Z_{s}\right)-\tilde{T}\left(\int_{0}^{\tilde{T}} Z_{s}^{-1} d Z_{s}\right)}{\left(\int_{0}^{\tilde{T}} Z_{s}^{-2} d s\right)\left(\int_{0}^{\tilde{T}} Z_{s}^{2} d s\right)-\tilde{T}^{2}} \\
&=\frac{\sigma^{2}}{2} \frac{\left(\int_{0}^{\tilde{T}} Z_{s}^{-2} d s\right)\left(\frac{1}{2}\left(Z_{\tilde{T}}^{2}-Z_{0}^{2}\right)-\tilde{T}\right)-\tilde{T} l o g\left[\frac{Z_{\tilde{T}}^{2}}{Z_{0}^{2}}\right]}{\left(\int_{0}^{\tilde{T}} Z_{s}^{-2} d s\right)\left(\int_{0}^{\tilde{T}} Z_{s}^{2} d s\right)-\tilde{T}^{2}} .
\end{aligned}
$$


Remark. Estimators $\hat{\alpha}_{1, T}, \hat{\alpha}_{2, T}$ and $\hat{\alpha}_{3, T}$ are equal, and so are estimators $\hat{\beta}_{1, T}, \hat{\beta}_{2, T}$ and $\hat{\beta}_{3, T}$, but $\hat{\alpha}_{1, T}, \hat{\alpha}_{3, T}, \hat{\beta}_{1, T}$ and $\hat{\beta}_{3, T}$ are obtained from the dynamics with additive noise.

\section{Stochastic Differential Equations of the Estimators of the Cox-Ingersoll-Ross Model}

In this section we consider the stochastic differential equation (2.19), which has been widely used to model interest rates in mathematical finance (see Cox, Ingersoll, \&Ross, 1985).

For the CIR model with $2 \alpha>\sigma^{2}$, there is a strictly positive ergodic solution of (2.19), as $T \rightarrow \infty$, whose distribution has gamma density $\Gamma\left(\frac{2 \alpha}{\sigma^{2}}, \frac{-2 \beta}{\sigma^{2}}\right)$.

By the ergodic theorem (see Kloeden and Platen, 1995) and equation (2.23) we obtain

$$
\begin{aligned}
& \frac{1}{\tilde{T}} \int_{0}^{\tilde{T}} Z_{s}^{-1} d s \longrightarrow \mathbf{E}\left(Z_{\infty}^{-\frac{1}{2}}\right) a . s . \\
& \frac{1}{\tilde{T}} \int_{0}^{\tilde{T}} Z_{s}^{-2} d s \longrightarrow \mathbf{E}\left(Z_{\infty}^{-1}\right) a . s . \\
& \frac{1}{\tilde{T}} \int_{0}^{\tilde{T}} Z_{s} d s \longrightarrow \mathbf{E}\left(Z_{\infty}^{\frac{1}{2}}\right) \text { a.s. } \\
& \frac{1}{\tilde{T}} \int_{0}^{\tilde{T}} Z_{s}^{2} d s \longrightarrow \mathbf{E}\left(Z_{\infty}\right) \text { a.s. }
\end{aligned}
$$

with $Z_{\infty}$ a random variable whose distribution is gamma, $\Gamma\left(\frac{2 \alpha}{\sigma^{2}}, \frac{-2 \beta}{\sigma^{2}}\right)$. The consistency and asymptotic normality of $\hat{\alpha}_{3, T}$ and $\hat{\beta}_{3, T}$ are obtained the same way as in Heyde (1997).

Our next result establishes the stochastic differential equations for the estimators of the parameters of the CIR model.

Theorem 1. Let the stochastic processes $\hat{\theta}_{1}=\left\{\hat{\theta}_{1, \tau} ; 0 \leq \tau<\tilde{T}\right\}$ and $\hat{\theta}_{2}=\left\{\hat{\theta}_{2, \tau} ; 0 \leq \tau<\tilde{T}\right\}$ be defined by

$$
\hat{\theta}_{1, \tau}=-\hat{\beta}_{3, \tau} \quad \text { and } \quad \hat{\theta}_{2, \tau}=-\frac{\hat{\alpha}_{3, \tau}}{\hat{\beta}_{3, \tau}}
$$

where $\hat{\alpha}_{3, \tau}$ and $\hat{\beta}_{3, \tau}$ are as in the example. 
Then

$d \hat{\theta}_{1, \tau}=-\left[\frac{\sigma^{2}}{2} \frac{H_{1}\left(\tau, Z_{\tau}\right)}{H_{2}\left(\tau, Z_{\tau}\right)}-\frac{\sigma^{2}}{4} \frac{H_{7}\left(\tau, Z_{\tau}\right)}{H_{2}\left(\tau, Z_{\tau}\right)}+\frac{H_{3}\left(\tau, Z_{\tau}\right)-H_{8}\left(\tau, Z_{\tau}\right)}{H_{2}\left(\tau, Z_{\tau}\right)} \hat{\theta}_{1, \tau}\right.$

$$
\left.+\frac{H_{7}\left(\tau, Z_{\tau}\right)}{H_{2}\left(\tau, Z_{\tau}\right)} \hat{\theta}_{1, \tau} \hat{\theta}_{2, \tau}\right] d \tau-\left[\frac{\sigma^{2}}{2} \frac{H_{4}\left(\tau, Z_{\tau}\right)}{H_{2}\left(\tau, Z_{\tau}\right)}\right] d \hat{W}_{\tau}
$$

$$
\begin{array}{r}
d \hat{\theta}_{2, \tau}=\quad\left[-\frac{\sigma^{4}}{4}\left(\frac{1}{H_{2}\left(\tau, Z_{\tau}\right)}-\tau \frac{H_{3}\left(\tau, Z_{\tau}\right)}{H_{2}\left(\tau, Z_{\tau}\right)}+\frac{H_{7}\left(\tau, Z_{\tau}\right)}{H_{2}\left(\tau, Z_{\tau}\right)}\right)-\frac{H_{8}\left(\tau, Z_{\tau}\right)}{H_{2}\left(\tau, Z_{\tau}\right)} \hat{\theta}_{1, \tau}\right. \\
(3.7)+\frac{H_{7}\left(\tau, Z_{\tau}\right)}{H_{2}\left(\tau, Z_{\tau}\right)} \hat{\theta}_{1, \tau} \hat{\theta}_{2, \tau}+\frac{\sigma^{2}}{2}\left(\frac{H_{5}\left(\tau, Z_{\tau}\right)}{H_{2}\left(\tau, Z_{\tau}\right)}+\frac{1}{2} \frac{H_{3}\left(\tau, Z_{\tau}\right)}{H_{2}\left(\tau, Z_{\tau}\right)}\right) \frac{1}{\hat{\theta}_{1, \tau}} \\
\left.+\frac{\sigma^{2}}{2} \frac{H_{1}\left(\tau, Z_{\tau}\right)}{H_{2}\left(\tau, Z_{\tau}\right)} \frac{\hat{\theta}_{2, \tau}}{\hat{\theta}_{1, \tau}}\right] d \tau-\left[\frac{\sigma^{2}}{2} \frac{H_{4}\left(\tau, Z_{\tau}\right)}{H_{2}\left(\tau, Z_{\tau}\right)}\right] d \hat{W}_{\tau}
\end{array}
$$

where

$$
\begin{aligned}
& H_{1}\left(\tau, Z_{\tau}\right)=Z_{\tau}^{-2}\left(\int_{0}^{\tau} Z_{s} d Z_{s}\right)-\int_{0}^{\tau} Z_{s}^{-1} d Z_{s} \\
& H_{2}\left(\tau, Z_{\tau}\right)=\left(\int_{0}^{\tau} Z_{s}^{-2} d s\right)\left(\int_{0}^{\tau} Z_{s}^{2} d s\right)-\tau^{2} \\
& H_{3}\left(\tau, Z_{\tau}\right)=Z_{\tau}^{2}\left(\int_{0}^{\tau} Z_{s}^{-2} d s\right)+Z_{s}^{-2}\left(\int_{0}^{\tau} Z_{s}^{2} d s\right)-2 \tau \\
& H_{4}\left(\tau, Z_{\tau}\right)=Z_{\tau}\left(\int_{0}^{\tau} Z_{s}^{-2} d s\right)-\tau Z_{\tau}^{-1} \\
& H_{5}\left(\tau, Z_{\tau}\right)=Z_{\tau}^{2}\left(\int_{0}^{\tau} Z_{s}^{-1} d Z_{s}\right)-\int_{0}^{\tau} Z_{s} d Z_{s} \\
& H_{6}\left(\tau, Z_{\tau}\right)=Z_{\tau}^{-1}\left(\int_{0}^{\tau} Z_{s}^{2} d s\right)-\tau Z_{\tau} \\
& H_{7}\left(\tau, Z_{\tau}\right)=\int_{0}^{\tau} Z_{s}^{-2} d s-\tau Z_{\tau}^{-2} \\
& H_{8}\left(\tau, Z_{\tau}\right)=Z_{\tau}^{2}\left(\int_{0}^{\tau} Z_{s}^{-2} d s\right)-\tau .
\end{aligned}
$$


Proof. According to (3.26) and (3.29), we may write

$$
\hat{\alpha}_{3, \tau}=\frac{\sigma^{2}}{2}\left(\frac{1}{2}+\frac{A_{\tau}}{B_{\tau}}\right)
$$

and

$$
\hat{\beta}_{3, \tau}=\frac{\sigma^{2}}{2} \frac{C_{\tau}}{B_{\tau}}
$$

where

$$
\begin{aligned}
A_{\tau} & =\left(\int_{0}^{\tau} Z_{s}^{2} d s\right)\left(\int_{0}^{\tau} Z_{s}^{-1} d Z_{s}\right)-\left(\int_{0}^{\tau} Z_{s} d Z_{s}\right) \tau, \\
B_{\tau} & =\left(\int_{0}^{\tau} Z_{s}^{-2} d s\right)\left(\int_{0}^{\tau} Z_{s}^{2} d s\right)-\tau^{2}, \\
C_{\tau} & =\left(\int_{0}^{\tau} Z_{s}^{-2} d s\right)\left(\int_{0}^{\tau} Z_{s} d Z_{s}\right)-\left(\int_{0}^{\tau} Z_{s}^{-1} d Z_{s}\right) \tau .
\end{aligned}
$$

Let $\hat{\alpha}_{\tau}$ and $\hat{\beta}_{\tau}$ denote the estimators $\hat{\alpha}_{3, \tau}$ and $\hat{\beta}_{3, \tau}$, respectively. Since $A, B$ and $C$ are semimartingales, we obtain the stochastic differential equations

$$
\begin{aligned}
& d \hat{\alpha}_{\tau}=\frac{\sigma^{2}}{2} \frac{1}{B_{\tau}} d A_{\tau}-\frac{\sigma^{2}}{2} \frac{A_{\tau}}{B_{\tau}^{2}} d B_{\tau}+d[A, B]_{\tau} \\
&= \frac{\sigma^{2}}{2} \frac{1}{B_{\tau}} d A_{\tau}-\left(\hat{\alpha}_{\tau}-\frac{\sigma^{2}}{4}\right) \frac{1}{B_{\tau}} d B_{\tau}+d[A, B]_{\tau}, \\
& d \hat{\beta}_{\tau}=\frac{\sigma^{2}}{2} \frac{1}{B_{\tau}} d C_{\tau}-\frac{\sigma^{2}}{2} \frac{C_{\tau}}{B_{\tau}^{2}} d B_{\tau}+d[C, B]_{\tau} \\
&=\frac{\sigma^{2}}{2} \frac{1}{B_{\tau}} d C_{\tau}-\hat{\beta}_{\tau} \frac{1}{B_{\tau}} d B_{\tau}+d[C, B]_{\tau},
\end{aligned}
$$

by Ito's formula (see Protter 1990, p. 74).

Here $[$,$] is the cross variation, which is also known as the bracket process.$ 
Moreover, $A$ and $C$ are semimartingales with continuous paths, and $B$ a semimartingale with paths of finite variation on compacts (the difference of two increasing processes). Then (see Protter 1990, p. 60)

$$
d[A, B]_{\tau}=d[C, B]=0 .
$$

Again, by Ito's formula and applying the latter argument to equations $(3.11)-(3.13)$,

$$
\begin{aligned}
d A_{\tau}= & \left(\int_{0}^{\tau} Z_{s}^{2} d s\right)\left(Z_{\tau}^{-1} d Z_{\tau}\right)+\left(Z_{\tau}^{2} d \tau\right)\left(\int_{0}^{\tau} Z_{s}^{-1} d Z_{s}\right) \\
& -\tau\left(Z_{\tau} d Z_{\tau}\right)-d \tau\left(\int_{0}^{\tau} Z_{s} d Z_{s}\right) \\
= & {\left[Z_{\tau}^{2}\left(\int_{0}^{\tau} Z_{s}^{-1} d Z_{s}\right)-\left(\int_{0}^{\tau} Z_{s} d Z_{s}\right)\right] d \tau } \\
& +\left[Z_{\tau}^{-1}\left(\int_{0}^{\tau} Z_{s}^{2} d s\right)-\tau Z_{\tau}\right] d Z_{\tau}
\end{aligned}
$$

$$
d B_{\tau}=\left(\int_{0}^{\tau} Z_{s}^{-2} d s\right)\left(Z_{\tau}^{2} d \tau\right)+\left(Z_{\tau}^{-2} d \tau\right)\left(\int_{0}^{\tau} Z_{s}^{2} d s\right)-2 \tau d \tau
$$

$$
\begin{aligned}
= & {\left[Z_{\tau}^{2}\left(\int_{0}^{\tau} Z_{s}^{-2} d s\right)+\left(\int_{0}^{\tau} Z_{s}^{2} d s\right) Z_{\tau}^{-2}-2 \tau\right] d \tau } \\
d C_{\tau}= & \left(\int_{0}^{\tau} Z_{s}^{-2} d s\right)\left(Z_{\tau} d Z_{\tau}\right)+\left(Z_{\tau}^{-2} d \tau\right)\left(\int_{0}^{\tau} Z_{s} d Z_{s}\right) \\
& -\tau\left(Z_{\tau}^{-1} d Z_{\tau}\right)-d \tau\left(\int_{0}^{\tau} Z_{s}^{-1} d Z_{s}\right), \\
= & {\left[Z_{\tau}^{-2}\left(\int_{0}^{\tau} Z_{s} d Z_{s}\right)-\left(\int_{0}^{\tau} Z_{s}^{-1} d Z_{s}\right)\right] d \tau } \\
& +\left[Z_{\tau}\left(\int_{0}^{\tau} Z_{s}^{-2} d s\right)-\tau Z_{\tau}^{-1}\right] d Z_{\tau} .
\end{aligned}
$$

The next two equations follow from the latter six

$$
d \hat{\alpha}_{\tau}=\left[\frac{\sigma^{2}}{2} \frac{Z_{\tau}^{2}\left(\int_{0}^{\tau} Z_{s}^{-1} d Z_{s}\right)-\left(\int_{0}^{\tau} Z_{s} d Z_{s}\right)}{\left(\int_{0}^{\tau} Z_{s}^{-2} d s\right)\left(\int_{0}^{\tau} Z_{s}^{2} d s\right)-\tau^{2}}\right.
$$




$$
\begin{array}{r}
\left.-\frac{Z_{\tau}^{2}\left(\int_{0}^{\tau} Z_{s}^{-2} d s\right)+\left(Z_{\tau}^{-2}\right)\left(\int_{0}^{\tau} Z_{s}^{2} d s\right)-2 \tau}{\left(\int_{0}^{\tau} Z_{s}^{-2} d s\right)\left(\int_{0}^{\tau} Z_{s}^{2} d s\right)-\tau^{2}}\left(\hat{\alpha}_{\tau}-\frac{\sigma^{2}}{4}\right)\right] d \tau \\
+\frac{\sigma^{2}}{2}\left[\frac{\left(Z_{\tau}^{-1}\right)\left(\int_{0}^{\tau} Z_{s}^{2} d s\right)-\tau Z_{\tau}}{\left(\int_{0}^{\tau} Z_{s}^{-2} d s\right)\left(\int_{0}^{\tau} Z_{s}^{2} d s\right)-\tau^{2}}\right] d Z_{\tau}
\end{array}
$$

$d \hat{\beta}_{\tau}=\left[\frac{\sigma^{2}}{2} \frac{Z_{\tau}^{-2}\left(\int_{0}^{\tau} Z_{s} d Z_{s}\right)-\left(\int_{0}^{\tau} Z_{s}^{-1} d Z_{s}\right)}{\left(\int_{0}^{\tau} Z_{s}^{-2} d s\right)\left(\int_{0}^{\tau} Z_{s}^{2} d s\right)-\tau^{2}}\right.$

$$
\begin{gathered}
\left.-\frac{Z_{\tau}^{2}\left(\int_{0}^{\tau} Z_{s}^{-2} d s\right)+\left(Z_{\tau}^{-2}\right)\left(\int_{0}^{\tau} Z_{s}^{2} d s\right)-2 \tau}{\left(\int_{0}^{\tau} Z_{s}^{-2} d s\right)\left(\int_{0}^{\tau} Z_{s}^{2} d s\right)-\tau^{2}} \hat{\beta}_{\tau}\right] d \tau \\
+\frac{\sigma^{2}}{2}\left[\frac{\left(Z_{\tau}\right)\left(\int_{0}^{\tau} Z_{s}^{-2} d s\right)-\tau Z_{\tau}^{-1}}{\left(\int_{0}^{\tau} Z_{s}^{-2} d s\right)\left(\int_{0}^{\tau} Z_{s}^{2} d s\right)-\tau^{2}}\right] d Z_{\tau} .
\end{gathered}
$$

Since

$$
\hat{\theta}_{1, \tau}=-\hat{\beta}_{\tau} \text { and } \quad \hat{\theta}_{2, \tau}=-\frac{\hat{\alpha}_{\tau}}{\hat{\beta}_{\tau}}
$$

by Ito's formula

$$
\begin{aligned}
& d \hat{\theta}_{1, \tau}=-d \hat{\beta}_{\tau} \\
& d \hat{\theta}_{2, \tau}=-\frac{1}{\hat{\beta}_{\tau}} d \hat{\alpha}_{\tau}-\hat{\theta}_{2, \tau} \frac{1}{\hat{\beta}_{\tau}} d \hat{\beta}_{\tau}-d[\hat{\alpha}, \hat{\beta}]_{\tau}
\end{aligned}
$$

and by (3.20)-(3.21) (see Protter 1990, p. 68),

$$
d[\hat{\alpha}, \hat{\beta}]_{\tau}=\frac{\sigma^{4}}{4}\left[\frac{1}{\left(\int_{0}^{\tau} Z_{s}^{-2} d s\right)\left(\int_{0}^{\tau} Z_{s}^{2} d s\right)-\tau^{2}}\right.
$$

$$
\left.-\tau \frac{Z_{\tau}^{2}\left(\int_{0}^{\tau} Z_{s}^{-2} d s\right)+Z_{\tau}^{-2}\left(\int_{0}^{\tau} Z_{s}^{2} d s\right)-2 \tau}{\left(\left(\int_{0}^{\tau} Z_{s}^{-2} d s\right)\left(\int_{0}^{\tau} Z_{s}^{2} d s\right)-\tau^{2}\right)^{2}}\right] d \tau,
$$


since the bracket process is bilinear.

Next, after some computations, we can verify that the second equation of (3.23) has the form

$$
\begin{aligned}
d \hat{\theta}_{2, \tau}= & -\frac{\sigma^{4}}{4}\left(\frac{1}{\left(\int_{0}^{\tau} Z_{s}^{-2} d s\right)\left(\int_{0}^{\tau} Z_{s}^{2} d s\right)-\tau^{2}}-\right. \\
& \left.-\tau \frac{Z_{\tau}^{2}\left(\int_{0}^{\tau} Z_{s}^{-2} d s\right)+Z_{\tau}^{-2}\left(\int_{0}^{\tau} Z_{s}^{2} d s\right)-2 \tau}{\left(\left(\int_{0}^{\tau} Z_{s}^{-2} d s\right)\left(\int_{0}^{\tau} Z_{s}^{2} d s\right)-\tau^{2}\right)^{2}}\right) \\
& +\frac{\sigma^{2}}{2}\left(\frac{Z_{\tau}^{2}\left(\int_{0}^{\tau} Z_{s}^{-1} d Z_{s}\right)-\int_{0}^{\tau} Z_{s} d Z_{s}}{\left(\int_{0}^{\tau} Z_{s}^{-2} d s\right)\left(\int_{0}^{\tau} Z_{s}^{2} d s\right)-\tau^{2}}\right. \\
& \left.+\frac{1}{2} \frac{Z_{\tau}^{2}\left(\int_{0}^{\tau} Z_{s}^{-2} d s\right)+Z_{\tau}^{-2}\left(\int_{0}^{\tau} Z_{s}^{2} d s\right)-2 \tau}{\left(\left(\int_{0}^{\tau} Z_{s}^{-2} d s\right)\left(\int_{0}^{\tau} Z_{s}^{2} d s\right)-\tau^{2}\right)^{2}}\right) \frac{1}{\hat{\theta}_{1, \tau}} \\
& +\frac{\sigma^{2}}{2}\left(\frac{Z_{\tau}^{-2}\left(\int_{0}^{\tau} Z_{s} d Z_{s}\right)-\int_{0}^{\tau} Z_{s}^{-1} d Z_{s}}{\left.\left(\int_{0}^{\tau} Z_{s}^{-2} d s\right)\left(\int_{0}^{\tau} Z_{s}^{2} d s\right)-\tau^{2}\right)} \frac{\hat{\theta}_{2, \tau}}{\hat{\theta}_{1, \tau}}\right] d \tau \\
& \left.+\left(\frac{Z_{\tau}\left(\int_{0}^{\tau} Z_{s}^{-2} d s\right)-\tau Z_{\tau}^{-1}}{\left(\int_{0}^{\tau} Z_{s}^{-2} d s\right)\left(\int_{0}^{\tau} Z_{s}^{2} d s\right)-\tau^{2}}\right) \frac{\sigma_{2, \tau}}{\hat{\theta}_{1, \tau}}\right] d Z_{\tau} \\
& \left.+\frac{\sigma_{\tau}^{-1}\left(\int_{0}^{\tau} Z_{s}^{2} d s\right)-\tau Z_{\tau}}{\left(\int_{0}^{\tau} Z_{s}^{-2} d s\right)\left(\int_{0}^{\tau} Z_{s}^{2} d s\right)-\tau^{2}}\right) \frac{1}{\hat{\theta}_{1, \tau}} \\
& \\
& +
\end{aligned}
$$

The proof of the theorem now follows substituting equation (2.23) in (3.25) and (3.21) and using the notation established in (3.8).

\section{Remarks}

In order to practically use the quasi-likelihood estimators $\hat{\alpha}_{i, T}$ and $\hat{\beta}_{i, T}$, for $i=1,2,3$ and $T>0$, obtained in Section 2 and the stochastic differential equations deduced in Section 3, it is necessary to make simulations 
of the dynamics associated to the estimators. The reader may refer to [2] for appropriate simulation schemes. The numerical data found in [6] may be used to check the quality of our estimators.

\section{References}

[1] Brown, R. H., Schaefer, S. M., The Term Structure of Real Interest Rates and the Cox, Ingersoll and Ross Model, J. of Financial Economics 35, pp. 3-42, (1994).

[2] Cox, J. S., Ingersoll, J. E., Ross, S. A., A theory of term structure of interest rates, Econometrica. 53, pp. 363-384, (1985).

[3] Feigin, P., Maximum likelihood estimation for continuous-time stochastic processes, J. Appl. Probab. 8, pp. 712-736, (1976).

[4] Heyde, C., Quasi-Likelihood and its Aplications, Springer, New York, (1997).

[5] Kloeden, P., Platen, E., Numerical Solution of Stochastic Differential Equations, Second Edition, Springer, Berlin, (1995).

[6] Kloeden, P., Platen, E., Shurz, H., Sorensen, M., On effects of discretization on estimators of drift parameters for diffusion processes, J. Appl. Probab. 33, pp. 1061-1076, (1996).

[7] Protter, P., Stochastic Integration and Differential Equations, Springer, New York, (1990).

\section{Eugenio Saavedra G.}

Departamento de Matemática y C. C.

Universidad de Santiago de Chile,

Casilla 307, Correo 2, Santiago,

Chile

e-mail : eugenio.saavedra@usach.cl 